\title{
Resultados tardios da operação de Heller associada à fundopli- catura no tratamento do megaesôfago: análise de 83 casos
}

\author{
F. A. M. Herbella, J . C. Del Grande, L.G. Lourenço, N.S. Mansur, C. M. Haddad
}

Trabalho realizado na Disciplina de Gastroenterol ogia Cirúrgica do Departamento de Cirurgia da Universidade F ederal de São Paulo - Escola Paulista de Medicina, São Paulo, SP

\begin{abstract}
RESUMO - ОвJ ETIVo. Estudo retrospectivo dos resultados tardios dos pacientes submetidos a miotomia e fundoplicatura para o tratamento do megaesôfago.

CASUísticA e métodos. Foram estudados 83 pacientes com seguimento de um a 186 meses (média de $40,0 \pm 47,4$ meses). A fundoplicatura em três planos, proposta por Pinotti, foi realizada em $15,7 \%$ dos doentes e a fundoplicatura parcial posterior em $83,1 \%$. A queixa pré-operatória principal foi disfagia, seguida de regurgitação e perda ponderal. A eti ologia chagási ca pode ser comprovada em $72,3 \%$ dos doentes, através de teste sorológico.

Resultados. No seguimento, $55,4 \%$ dos doentes estavam assintomáticos, $34,9 \%$ queixavam-se de disfagia esporádica, $14,4 \%$ de pirose, $8,4 \%$ de regurgi tação e $2,4 \%$ de di sfagi a igual pré-operatória, sendo esses últimos re-operados, com melho-
\end{abstract}

\section{NTRODUÇÃO}

O megaesôfago é uma das manifestações digestivas da doença de Chagas $^{1}$ e, a despeito do grande número de casos operados em diversos centros, as dificuldades de acompanhamento desses doentes impedem freqüentemente a realização de trabaI hos prospectivos e com segui mento pós-operatório a longo prazo. Por outro lado, deve-se, também, lembrar que com o advento da via de acesso por vídeo-laparoscopia, e os bons resultados obtidos por esse método², aumenta o interesse dos estudos comparativos a longo prazo entre essas duas vias de acesso.

Apresentamos neste artigo a evolução tardia, em estudo retrospectivo, de 83 doentes portadores de megaesôfago, submetidos à operação de Heller associado à fundoplicatura, na qual a via de acesso foi laparotomia.

\section{CASUÍSTICA}

Nossa casuística consta de 83 pacientes (42 homens, 41 mul heres) operados entre 1977 ejunho ra da sintomatologia. R efluxo gastro-esofági co foi constatado em $\mathbf{8 , 4} \%$ dos doentes. Outras complicações tardias foram: hérnia paraesofágica, deslizamento da fundoplicatura, esôfago de Barrett e neoplasia do esôfago.

Conclusões. Os autores salientam a necessidade de acompanhamento clínico e endoscópico perene dos doentes, mesmo operados, devido à possibilidade de complicações tardias, em especial o câncer. Concluem por bons resultados tardios em relação à disfagia. Propõem, como alternativa à esofagectomia, a operação de Heller em pacientes portadores de megaesôfago grau IV com más condições clínicas, que não suportariam uma cirurgia de maior porte.

Unitermos: Acalásia esofágica. Cardiomiotomia. Cirurgia. Resultados.

de 1995, cuja idade média era de 43,2 \pm 14,2 anos (variação de 79-17 anos).

O sintoma principal foi disfagia, presente em 82 $(98,8 \%)$ paci entes, e o tempo médio de queixa era de $8,5 \pm 8,8$ anos (30 dias - 30 anos). Regurgitação foi referida por $63(75,9 \%)$ doentes. Perda ponderal estava presente em $55(66,3 \%)$ pacientes, com média de $1,37 \pm 1,76(0,05-7,75) \mathrm{kg} / \mathrm{mês}$.

Outras manifestações da doença de Chagas e outras mol éstias estavam associadas em 49 (59,0\%) doentes, sendo representadas por: cardiopatia, em 38 (45,8\%), megacólon, em 20 (24,1\%), colecistite crônica calcul osa, em cinco $(6,0 \%)$, nefropatia crônica avançada, em dois $(2,4 \%)$, hérnia hiatal, em dois $(2,4 \%)$, hérnia inguinal, em um $(1,2 \%)$, divertícul o de Zenker, em um (1,2\%), acalásia do piloro, em um (1,2\%), hipertensão portal, em um (1,2\%) e tuberculose peritoneal, em um (1,2\%).

Dois doentes $(2,4 \%)$ haviam sido previamente submeti dos à operação de Heller e fundoplicatura em outros serviços. A etiologia chagásica foi comprovada através do teste sorológi co de Machado e Guerreiro em 60 (72,3\%) doentes. O exame radiológico, realizado em todos os pacientes, permitiu 


\begin{tabular}{|cc|}
\hline Tabela I - Tempo de seguimento. \\
\hline Tempo de Seguimento & \% de doentes \\
$<1$ ano & 41,5 \\
$1-2$ anos & 11,0 \\
$2-3$ anos & 8,5 \\
3-4 anos & 6,1 \\
$4-5$ anos & 7,3 \\
$>5$ anos & 25,6 \\
\hline
\end{tabular}

dividi-los, segundo a classificação de Rezende, em portadores de megaesôfago grau I (2,4\%), II (36,1\%), III $(48,2 \%)$ e IV (13,2\%). O exame endoscópico, realizado em $81(97,6 \%)$ pacientes, mostrou dilatação esofagiana, em 67 (80,7\%), estase, em 53 $(60,9 \%)$, dificuldade de abertura e transposi ção do esfíncter esofágico inferior, em 49 (59,0\%) e esofagite, em 26 (31,3\%). Outros achados foram: hérnia hiatal $(4,8 \%)$, refluxo gastro-esofágico $(3,6 \%)$, divertículo de Zenker (1,2\%), varizes esofagianas $(1,2 \%)$ e dificul dade de abertura e transposição do piloro (1,2\%). Três pacientes (3,6\%) mostraram exame endoscópico normal.

Nenhum doente foi submetido previamente a dilatações endoscópicas.

\section{MÉ TODO}

A via de acesso utilizada foi laparotomia mediana supra-umbilical . A cardiomiotomia foi realizada por uma extensão média de 9,0 $\pm 1,7(4-13) \mathrm{cm}$, sendo 6,6 \pm 1,5 (3-11) cm no esôfago e 2,3 \pm 1,0 (15) cm no estômago. Após a miotomia praticou-se sempre fundoplicatura, a qual entre 1977 e 1991 constou da fundoplicatura posterior, com pontos ancorados às margens da miotomia (técnica de Lind), que foi praticada em $69(83,1 \%)$ pacientes. Desde 1991, passou-se a praticar fundoplicatura em três planos ${ }^{3,4}$, realizada em $13(15,7 \%)$ pacientes. Um paciente $(1,2 \%)$ foi submetido à fundoplicatura anterior ${ }^{5}$. A extensão média das fundoplicaturas foi de 4,7 $\pm 1,6(2-6) \mathrm{cm}$. N os doentes com colecistopatia crônica calculosa realizou-se simultaneamente col ecistectomia e exploração radi ol ógica das vias biliares, nos portadores de hérnia hiatal praticou-se a hiatoplastia, nos casos de megacól on associado (cinco doentes) realizou-se a correção dessa doença através de sigmoidectomia e anurretomiectomia, e no doente com de divertículo de Zenker, realizou-se a diverticulectomia. Os demais doentes com megacólon associado obtiveram meIhora da obstipação com tratamento clínico e foram encaminhados ao grupo de coloproctologia.

Os dados estatí sti cos for am avali ados pel o testet de Student e Qui-quadrado, com significância para $p<0,05$.

\section{RESULTADOS}

Acidentes intra-operatórios ocorreram em 15 $(18,1 \%)$ pacientes, sendo representados por lesão do baço em oito $(9,6 \%)$, perfuração da mucosa esofági ca em sete $(8,4 \%)$ e lesão do fígado em um $(1,2 \%)$, sendo que um paciente apresentou lesão da mucosa e do baço.

O tempo de seguimento foi de 40,0 $\pm 47,4$ (1-186) meses (tabela I). Quarenta e seis pacientes $(55,4 \%)$ apresentavam-se assintomáticos no seguimento. Disfagia esporádi ca era queixa de 29 (34,9\%) doentes, pirose de 12 (14,4\%), regurgitação de sete $(8,4 \%)$, quatro $(4,8 \%)$ pacientes relataram perda de peso, e dois $(2,4 \%)$ queixavam-se de disfagia igual ao pré-operatório.

A avaliação radiológica, realizada em todos os doentes, após a primeira consulta no pós-operatório, ou em caso de sintomas importantes, mostrou refluxo gastroesofágico, em quatro $(4,8 \%)$ pacientes, deslizamento da fundoplicatura para o tórax, em um $(1,2 \%)$ e hérnia paraesofágica, em um (1,2\%). A avaliação endoscópica, realizada em todos os doentes após a primeira consulta no pósoperatório, anualmente ou em casos de sintomas importantes, revelou esofagite, em 13 (15,7\%) pacientes, refluxo gastroesofágico, em três $(3,6 \%)$, esôfago de Barrett, em dois (2,4\%) e neoplasia de esôfago, em dois (2,4\%). A manometria esofágica foi utilizada em apenas pequena porcentagem dos doentes, pois não dispúnhamos desta no início de nossa experiência, sendo que seus resultados não expressariam significantemente nossa casuística, portanto, não serão comentados.

Sete $(8,4 \%)$ pacientes foram re-operados: um $(1,2 \%)$ em conseqüência de refluxo gastroesofágico intenso e esôfago de Barrett, sendo submetido a fundoplicatura total, dois (2,4\%), devido à disfagia persistente importante, foram submetidos, um à operação de Merendino, outro à nova miotomia e fundoplicatura e três (3,6\%) foram esofagectomizados por haver ocorrência de neoplasia do esôfago em dois (2,4\%) e hérnia paraesofági ca em um $(1,2 \%)$.

$\mathrm{N}$ ão houve mortalidade tardia relacionada ao ato operatório.

\section{DISCUSSÃO}

O seguimento dos portadores de megaesôfago, mesmo os operados, deve ser perene, devido à possibilidade de ocorrência de complicações tar di as importantes, em especial o câncer. Em nossa casuística, os pacientes foram examinados pelo menos uma vez após o primeiro mês de pós-operatório, não só porque a maior parte das complica- 


\begin{tabular}{|c|c|c|c|c|c|}
\hline & $\begin{array}{l}\text { Disfatia } \\
\text { P.O. }\end{array}$ & $\begin{array}{c}\text { Pirose } \\
\text { P.O. }\end{array}$ & $\begin{array}{c}\text { Regurgitação } \\
\text { P.O. }\end{array}$ & $\begin{array}{c}\text { R. G. E. } \\
1\end{array}$ & Total \\
\hline Idade (anos) & $41,0 \pm 12,2$ & $47,9 \pm 13,9$ & $33,7 \pm 12,3$ & $41,1 \pm 7,2$ & $43,2 \pm 14,2$ \\
\hline Sexo masculino (\%) & 13,2 & 2,4 & 2,4 & 2,4 & 50,6 \\
\hline Sexo feminino (\%) & $21,7^{*}$ & 12,0 * & 6,0 & 6,0 & 49,4 \\
\hline Tempo de disfagia (anos) & $9,9 \pm 9,6$ & $10,7 \pm 13,3$ & $5,5 \pm 4,5$ & $7,1 \pm 7,4$ & $8,5 \pm 8,8$ \\
\hline Queixa de perda de peso (\%) & 20,5 & 12,0 & 6,0 & $0 \%$ & 66,3 \\
\hline Miotomia esôfago $(\mathrm{cm})$ & $6,7 \pm 1,5$ & $6,2 \pm 1,3$ & $5,6 \pm 1,4$ & $7,1 \pm 2,5$ & $6,6 \pm 1,5$ \\
\hline Miotomia estômago (cm) & $2,3 \pm 0,9$ & $2,0 \pm 0,7$ & $2,0 \pm 0,8$ & $2,1 \pm 0,4$ & $2,3 \pm 1,0$ \\
\hline Lesão mucosa esofágica (\%) & 2,4 & 1,2 & 0,0 & 1,2 & 8,4 \\
\hline Fundoplicatura a Lind (\%) & 30,1 & 13,2 & 8,4 & 7,2 & 83,1 \\
\hline Fundoplicatura a Pinotti (\%) & 4,8 & 1,2 & 0,0 & 1,2 & 15,7 \\
\hline Extensão da fundoplicatura $(\mathrm{cm})$ & $4,3 \pm 1,1$ & $5,4 \pm 2,3$ & $4,0 \pm 0,0$ * & $2,1 \pm 0,4$ & $4,7 \pm 1,6$ \\
\hline Grau I (\%) & 1,2 & 50 & 50 & 1,2 & 2,4 \\
\hline Grau II (\%) & 22,9 & 0,2 & 0,1 & 4,8 & 36,1 \\
\hline Grau III (\%) & 18,0 & 0,1 & 0,1 & 2,4 & 48,2 \\
\hline Grau IV (\%) & 2,4 & 0,1 & 0,1 & 0,0 & 13,2 \\
\hline Ganho de peso pós-operat. (\%) & N.A & N.A. & N. A. & 14,3 & 21,7 \\
\hline \multicolumn{6}{|c|}{$\begin{array}{l}\text { 1- Refluxo Gastro-esofágico pós-operatório } \\
\text { * - Estatisticamente significante } \\
\text { N. A. - Não Avaliado } \\
\text { P.O. - Pós-Operatório }\end{array}$} \\
\hline
\end{tabular}

ções tardias já se manifestam nos primeiros 30 dias pós-operatórios, como também pela dificuldade no acompanhamento a longo prazo desses doentes. Mesmo adotando esse critério, dos 104 doentes por nós operados 6 , apenas 83 foram acompanhados por pelo menos um mês e incluídos nessa casuística, mostrando a dificuldade no seguimento desses doentes, os quais muitas vezes retornam a seus estados de origem após o tratamento. Outro ponto a ser destacado é que esse é um estudo retrospectivo, como a maioria dos trabal hos publicados sobre megaesôfago ${ }^{7-9}$, o que demonstra a dificul dade de seguimento também referida por outros autores. Deve ser destacado que, a despei to de todas as dificuldades, pudemos seguir $25 \%$ dos doentes por período superior a cinco anos. Acreditamos que a inclusão de dois doentes previamente operados não prejudica os resultados, pois, além de perfazerem um pequeno percentual dos casos, os resultados são pertinentes a cirurgia de Heller e fundoplicatura, exatamente o procedimento a que foram submetidos.

No pós-operatório da mai oria dos pacientes portadores de megaesôfago, submetidos a miotomia e fundoplicatura, pode ocorrer algum grau de disfagia, porém essa é geralmente leve, esporádica e de menor intensidade que a pré-operatória, podendo ser explicada, em parte, pela desperistalse esofágica que persiste mesmo após a operação, assim sendo, necessitam apenas de orientação higiêno-dietética. Não encontramos significância estatística que pudesse relacionar a disfagia pós- operatória leve em rel ação a idade, tempo de queixa pré-operatória de disfagia, perda de peso, extensão proximal e distal da miotomia, tipo de fundoplicatura e lesão intra-operatória da mucosa esofágica. Encontramos, entretanto, com significância, preponderância da disfagia pós-operatória leve entre as mulheres $(45,2 \%)$ comparadas aos homens $(25,6 \%)$, fato não descrito na literatura e para o qual não encontramos justificativa aceitável .

Disfagia intensa e persistente, desde o pósoperatório imediato geralmente, está associada a fal ha técni ca na confecção da mi otomia e/ou fundoplicatura, desde que a operação tenha sido indicada corretamente $e^{3,4,10,11}$. Nessas condições, suspeita-se de miotomia insuficiente e/ou fundoplicatura real izada sob tensão, o que dificul ta o esvaziamento do esôfago. J á disfagia de aparecimento tardi o rel aci ona-se a reaproximação das bordas da miotomia, fibroselocal ou, ai nda, devi do ao refl uxo gastro-esofágico com esofagite estenosante, que pode advir como complicação dessa cirurgia. Em nossos casos, disfagia manteve-se inalterada em dois $(2,4 \%)$ doentes, sendo ambos re-operados. U m deles, portador de megaesôfago grau IV, foi submetido a nova cardiomiotomia após 22 meses da operação inicial, tornando-se assintomático. O outro, foi submetido a oper ação de Merendino, com boa evolução, falecendo por outras causas quatro anos após.

A maioria dos autores $3,7,10,12-14$ apresenta seus resultados finais quanto à disfagia baseados na classificação proposta por Vantrapen e Hellemans ${ }^{15}$, 
sendo que a grande mai oria mostra bons resultados acima de $90 \%^{1,3,4,7,8,10,14,16}$, o que ocorreu também em nossa casuística, já que atingiu $97,6 \%$ dos casos.

A pirose tem sido citada como sintoma pósoperatório da cirurgia de Heller em porcentagens que variam de $0 \%$ a $25 \%$ 7,9,17. Em nossa casuística observamos que 12 (14,4\%) doentes apresentavam essa queixa com freqüência, com incidência significativamente maior nas mulheres $(24,4 \%)$, quando comparada aos homens (4,8\%). A pirose pode representar manifestação clínica do refluxo gastroesofágico e, na tentativa de comprová-lo, empregamos os exames endoscópico e radiológico . Pudemos, então, observar que em somente um doente foi possível, por esses métodos propedêuticos, a demonstração de refluxo. A destruição do esfíncter esofági co inferior e das fibras oblíquas do estômago são vistas, atualmente, como as principais causas do refluxo gastroesofágico. N esses doentes ${ }^{11}$, por esse motivo, a miotomia à Heller tem sido associada à fundoplicatura, a qual visa, justamente, impedir o refluxo. Em nossos pacientes utilizávamos a fundoplicatura à Lind, porém devido a ocorrência de casos de refluxo optamos a partir de 1991 pel a fundoplicatura em três planos, que, entretanto, mostrou a mesma taxa de refluxo que a opção prévia . Os índices de refluxo gastroesofágico nesse tipo de operação variam de $0 \%$ a $35,2 \% 10,12,13,16,17,18$. E m nossa casuística, observamos que, além dos doentes com queixa de pirose, em outros seis $(8,4 \%)$, assintomáticos em relação ao refluxo, foi comprovado existência de refluxo gastroesofágico, o que demonstra a necessidade de acompanhamento clínico e com exames mesmo em pacientes assintomáticos. Tal fato fica mais evidente com a constatação que dois $(2,4 \%)$ dos nossos doentes assintomáticos apresentavam esôfago de Barrett. Alguns autores ${ }^{8,19}$ preconizam a mi otomia curta, sem atingir o estômago, o que garantiria a integridade parcial do esfíncter e eliminaria a necessidade de realização de válvula anti-refluxo, mesmo correndo o risco de persistência da disfagia ${ }^{19}$. O mesmo ocorre com as fundoplicaturas totais, que exibem menores índices de refluxo, porém piores resultados frente à disfagi $a^{17}$. Não houve maior incidência de refluxo quando analisada a idade dos doentes, sexo, grau radiológico do megaesôfago, extensão proximal e distal da miotomia, tipo de fundoplicatura e sua extensão e lesão da mucosa esofágica.

Em relação à regurgitação o resultado por nós observado com a realização da miotomia e fundoplicatura também pode ser considerado satisfatório, já que dos $63(75,4 \%)$ doentes que apresenta- vam a queixa apenas seis (7,2\%) persistiram com o sintoma no pós-operatório. Deve-se, porém, assinalar que um $(1,2 \%)$ doente que não referia regurgitação no pré-operatório passou a apresentar a queixa após a cirurgia, fato esse para o qual não encontramos explicação plausível. A regurgitação pode advir de miotomia incompleta, fal ha técnica na elaboração da fundoplicatura, ambos associados a desperistalse esofágica ou, ainda, indicação incorreta do procedimento. Procurando correlacionar a presença do sintoma com falha técnica, pudemos observar que, estranhamente para nós a regurgitação pós-operatória em nossa casuística estava associada a uma menor extensão de fundoplicatura.

A neoplasia do esôfago pode ocorrer nos portadores de megaesôfago, provavel mente em razão da estase crônica e conseqüentes al terações epiteliais displásicas, fato que obriga o seguimento endoscópico periódico desses doentes. No paciente submetido a fundoplicatura e miotomia também esse seguimento deve ser regra, pois a ocorrência de câncer também está descrita nesses doentes 3,7,11,16,19, como pudemos observar em nossa casuística, já que dois $(2,4 \%)$ doentes desenvolveram câncer do esôfago na evolução pós-operatória, dois e quatro anos após a cirurgia. O uso do lugol é descrito como forma de detecção precoce da neoplasia, porém, não foi empregado em nenhum de nossos casos.

A hérnia paraesofágica pós-operatória é também descrita por outros autores ${ }^{10}$, ocorrendo, possivelmente, por manipulação excessiva do hiato esofágico no ato cirúrgico; não aproximação no intra-operatório dos braços do hiato previamente alargado ou redução do calibre do órgão após a cirurgia. O deslizamento da fundoplicatura para o tórax, presente em um de nossos doentes, pode ter as mesmas explicações para sua ocorrência.

Contrariamente ao fr eqüentemente proposto na literatura ${ }^{4,20}$, tratamos com mi otomi a onze doentes com megaesôfago de grau avançado (IV). A indicação de mi otomia nesse grupo de doentes tem justificativa no fato de não indicarmos esofagectomia em megaesôfago em nosso serviço até 1983 (três pacientes) ou pela associação de enfermidades graves, três pacientes eram portadores de megacólon e, devido à colectomia concomitante, optouse pela cirurgia de Heller; três eram portadores de desnutrição protêico-calórica de grau III e cardiopatia, sendo um deles ainda tuberculoso; um cursava com hi pertensão portal e o último com tuberculose pulmonar. Esses pacientes mostraram boa evolução clínica, com dois doentes queixando-se de disfagia ocasional, um de regurgitação e um de pirose, não havendo significância estatística quan- 
do comparado aos graus não avançados. Outras complicações tardias aconteceram em apenas um dos paci entes que apresentou esôfago de Bar rett. $O$ tempo de seguimento médio desses pacientes foi de $52,2 \pm 30,8$ (dois meses - nove anos) meses.

\section{CONCLUSÕES}

Observando nossos resultados, concluímos que há grande dificuldade no seguimento dos doentes portadores de megaesôfago, que, contudo, a despeito das dificuldades, deve ser perene e com auxíli o de propedêutica armada, já que há a possibilidade da ocorrência de complicações tardias, especialmente o câncer. Cardiomiotomia associada a fundoplicatura é a cirurgia de escol ha pela maior parte dos cirurgiões para o tratamento do megaesôfago e mostrou-se uma operação segura e com bons resultados, porém, ainda com complicações dependentes da técnica cirúrgica. Apesar do seguimento menor, não houve diferença nos resultados comparando os dois tipos de fundoplicatura (Lind e Pinotti).

Contrariamente ao freqüentemente proposto na literatura, tratamos com a cirurgia de Heller 11 doentes portadores de megaesôfago avançado (grau IV), que apresentaram boa evolução, constituindose em al ternativa para o tratamento de doentes sem condições clínicas para uma cirurgia de maior porte como a esofagectomia.

\section{SUMMARY}

\section{Late Results of Heller operation and fundo- plication for the treatment of the megaeso- phagus - analysis of 83 cases}

BACKGROUND. Retrospective study of the late results of the Heller's cardiomyotomy and fundoplication for the treatment of the megaesophagus.

Material and Methods. Were studied 83 patients with a follow-up from one to 186 months (average 40.0 \pm 47.4 months). The fundoplications used were in three plans in $15.7 \%$ and posterior in $83.1 \%$. The main pre-operatory complain was disphagia followed by regurgitation and loss of weight. Chagas, Disease as the etiology was confirmed in $72.3 \%$ of the patients.

RESULTS. In the follow-up $55.4 \%$ of the patients were assymptomatic, $34.9 \%$ complained of sporadic dysphagia, $14.4 \%$ of heartburn, $8.4 \%$ of regurgitation and $2.4 \%$ did not changed the dysphagia, these being re-operated and had improvement the symptoms. Gastro-esophageal reflux was noted in $8.4 \%$ of the pati ents. Other late complications were par-esophageal hernia, sliping of the fundoplication, Barrett esophagus and cancer.

Conclusions. The necessity of a long-term clinical and endoscopical follow-up, even after surgery, owing to the possibility of late complications, especially cancer was emphasized. The lateresults are good in relation to the dysphagia. Myotomy is proposed as an alternative to patients with advanced megaesophagus with bad clinical conditions who can not be submitted to an esophagectomy. [Rev Ass Med Bras 1999; 45(4): 317-22]

KEY WORDS: Esophageal achalasia. Cardiomyotomy. Surgery. Results.

\section{REFERÊNCIAS BIBLIOGRÁFICAS}

1. Pinotti HW, Felix VN, Zilberstein B. Surgical complications of Chaga's disease: megaesophagus, achalasia of the pylorus, and cholelithiasis. World J Surg 1991; 15: 198-204.

2. Domene CE. Cardiomiectomia com fundoplicatura parcial videol aparoscópica no tratamento do megaesôfago não avançado. Faculdade de Medicina da Universidade de São Paulo 1996; Tese de Livre-Docência.

3. Pinotti HW, Ellenbogen G, Rodrigues J G, Raia A. Surgical treatment of the megaesophagus. Chir Gastroent 1977; 11 (1): 7-14.

4. Pinotti HW, Sakai P, I shioka S. Cardiomyotomy and fundoplication for esophageal achalasia. J pn. J. Surg. 1983; 13 (5): 399-403.

5. J ekler J, Lhotka J. Modified Heller procedure to prevent postoperative reflux esophagitis in patients with achalasia. AmJ Surg 1967; 113: 251-254.

6. Del Grande J C, Herbella FAM, Lourenço LG, Mansur NS, Haddad CM. Complicações operatórias eresultados imediatos da cardiomiotomia com fundoplicatura no tratamento do megaesôfago - análise de 104 casos. GED-Gastroenterol ogia e Endoscopia Digestiva 1996; 15 (5): 156 - 160.

7. Moreno González E, Garcia Alvarez A, Landa Garcia I, Gómez Gutierrez M, RicoSelas P, Garcia Garcia I , J over Navalón J M, Arias Diaz J. Results of surgical treatment of esophageal achalasia. Multicenter retrospectivestudy of 1,856 cases. Int. Surg. 1988; 73: 69-77.

8. Mattioli S, Di Simone MP, Bassi F, Pilotti V, FeliceV, Pastina M, Lazzari A, Gozzetti G. Surgery for esophageal achalasia. Long term results with three different techniques. HepatoGastroenterol 1996; 43: 492-500.

9. Picciocchi A, Cardillo G, D’U go D, Castrucci G, Mascellari L, Granone P. Surgical treatment of achalasia: a retrospective comparative study. J pn J Surg 1993; 23: 855-859.

10. Bonavina L, Nosadini A, Bardini R, Baessato M, Peracchia A. Primary treatment of esophageal achalasia. Arch Surg 1992; 127: 222-27.

11. Csendes A. Results of surgical treatment of achalasia of the esophagus. Hepato-Gastroenterol 1991; 31: 474-80.

12. Andreollo NA, Earlam RJ . Heller's myotomy for achalasia: is an added anti-reflux procedurenecessary? BrJ Surg 1987; 74: 765-69.

13. Malthaner RA, Todd TR, Miller L, Pearson FG. Long-term results in surgically managed esophageal achalasia. Ann Thorac Surg 1994; 58: 1.343-47. 
14. Parrilla Paricio P, Martínez de Haro L, Ortiz A, Aguayo J L. Achalasia of the cardia: Iong-term results of oesophagomyotomy and posterior partial fundoplication. Br J Surg 1990; 77: 1.371-74.

15. Vantrapen G, Hellemans J. Treatment of achalasia and related motor disorders. Gastroenterology 1980; 79: 144-54.

16. Ribet $M$, Bonnet $P$, Pruvot $F R$, Mensier $E$. L'opération de Heller pour méga-oesophage techniques et résultats. Lyon Chir 1990; 86 (1): 9-11.

17. Stipa S, Fegiz G, Iascone C, Paolini A, Moraldi A, De Marchi C,
Chieco PA. Heller-Belsey and Heller-Nissen operations for achalasia of theesophagus. Surg Gynecol Obstet 1990; 170: 212-16.

18. J amieson GG. Gastro-esophageal reflux following myotomy for achalasia. Hepato-Gastroenterol 1991; 38: 506-509.

19. Ellis FH. Oesophagomyotomy for achalasia: a 22-year experience. Br J Surg 1993; 80: 822-85.

20. Aquino J LB. Tratamento do megaesôfago pel a mucosectomia com conservação da túnica muscular esofágica por via cervicoabdominal. Universidadeestadual de Campinas 1996; Tese de Doutorado. 